\title{
Orbital Picture in Molecular Inner-Shell Excited States of Rydberg-Valence Mixed Character
}

\author{
Nobuhiro Kosugi \\ UVSOR, Institute for Molecular Science, Myodaiji, Okazaki 444-8585, Japan
}

Received on 14 January, 2005

\begin{abstract}
The core-to- $\sigma^{*}$ excited state is repulsive for the bond elongation; on the other hand, the core ionized state and the core-to- $\pi^{*}$ state are bound and the core-to-Rydberg states are almost parallel to the potential energy curve of the core ionized state. Thus, the core-electron excitation to the unoccupied $\sigma^{*}$ orbital can be mixed with the one-electron Rydberg or continuum orbital, as dependent on the bond distance, and even with the unoccupied $\pi^{*}$ orbital in some cases. Within the framework of one-electron picture, we show $\sigma^{*}$ orbitals mixed with Rydberg character in the $1 \mathrm{~s}$ excitation of $\mathrm{O}_{2}$ and $\mathrm{CH}_{3} \mathrm{~F}$, and Rydberg orbitals mixed with valence character in the $1 \mathrm{~s}$ excitation of $\mathrm{CH}_{4}, \mathrm{CO}_{2}$, and $\mathrm{N}_{2} \mathrm{O}$.
\end{abstract}

\section{INTRODUCTION}

The atomic and molecular core-to-Rydberg excitation is converging to a certain core ionization threshold $\left(\mathrm{E}_{t h}\right)$. In addition, most molecules have unoccupied molecular orbitals of anti-bonding valence character such as $\sigma^{*}$ and $\pi^{*}$. These antibonding orbitals can also accept an excited electron from the inner shell. The potential energy curve of the core-to- $\sigma^{*}$ valence excited state [1] is repulsive for a specified $\sigma$ bond; then, the core-to- $\sigma^{*}$ excited state is lower than the continuum and Rydberg region at the longer bond distance and is embedded in the continuum at the shorter distance. That is, two kinds of interaction involving the valence state should be discussed along the potential energy curve for a specified $\sigma$ bond[1]: the Rydberg-valence interaction below $\mathrm{E}_{t h}$ and the continuumvalence interaction above $\mathrm{E}_{t h}$. In the present work, we discuss the former interaction within one-electron picture and show how the valence and/or Rydberg orbitals look like through the interactions.

\section{RYDBERG CONTRIBUTION IN CORE-TO- $\sigma^{*}$ EXCITATION}

\section{A. $\mathbf{O}_{2}$}

$\mathrm{N}_{2}$ has a triple covalent bond and the $3 \sigma_{u} *$ molecular orbital is of strong antibonding character; then, the N1s excitation to this $\sigma^{*}$ orbital is observed above $\mathrm{E}_{t h}$ as a well-known shape resonance. In molecules having weak covalent bonds, the $1 \mathrm{~s} \rightarrow \sigma^{*}$ excited state is possibly observed below $\mathrm{E}_{t h}$. We can expect Rydberg-valence (RV) mixings in the case of the same p-symmetry as the $2 \mathrm{p} \sigma^{*}$ orbital. As already discussed $[1,2]$, the strong RV mixing results in new potential energy curves due to the avoided curve crossing of the Rydberg ( $\mathrm{p}$ type) and valence $\left(2 \mathrm{p} \sigma^{*}\right)$ states.

The Rydberg features in the $\mathrm{O} 1 \mathrm{~s}$ excitation spectrum of $\mathrm{O}_{2}$ with a triplet ground state $\left({ }^{3} \Sigma_{g}^{+}\right)$are very complicated due to two ionization thresholds, ${ }^{4} \Sigma^{+}$and ${ }^{2} \Sigma^{+}$[2,3]. The angle-resolved photoion spectroscopy (ARPIS) has shown that the core-to- $\sigma^{*}\left(3 \sigma_{u} *\right)$ excited states give exchange-split two strong resonances in the Rydberg region [2,3] and that the ${ }^{4} \Sigma^{+}$channel gives vibrational enhancements in the $3 \mathrm{p \sigma}$ Rydberg state and the ${ }^{2} \Sigma^{+}$channel has no distinct evidence for the RV mixing [2]. Fig. 1 shows (a) this $3 \sigma_{u}^{*}$ orbital before the RV mixing and (b) the RV-mixed $3 p \sigma-3 \sigma_{u} *$ orbital in the Franck-Condon region. This kind of the RV mixing is not effective for the s-type or d-type Rydberg states with different symmetry from the $2 \mathrm{p} \sigma^{*}$ orbital.

(a)

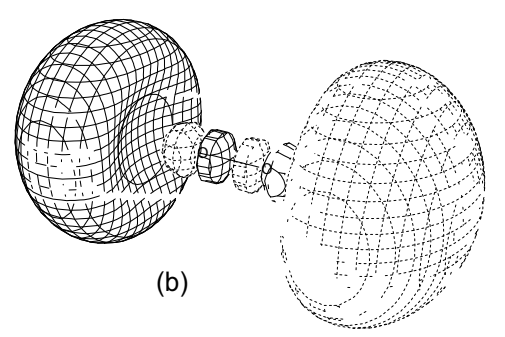

FIG. 1: Contour maps of (a) the $3 \sigma_{u} *$ orbital obtained before the RV mixing (without any diffuse function) and (b) the RV-mixed $3 p \sigma-$ $3 \sigma_{u} *$ orbital in the $\mathrm{O} 1 \mathrm{~s}$ excitation of $\mathrm{O}_{2}$.

\section{B. $\mathrm{CH}_{3} \mathrm{~F}$}

In $\mathrm{N}_{2}$, the $\mathrm{N} 1 \mathrm{~s}$ excitation to the $\pi^{*}$ orbital is located lower than the Rydberg region. Even in saturated molecules, the excitation to the $\sigma^{*}$ orbital is possibly located below the Rydberg region in the case of very weak $\sigma$ covalent bonds. In $\mathrm{CH}_{3} \mathrm{~F}$ with a very weak $\sigma$ covalent bond between $\mathrm{CH}_{3}$ and $\mathrm{F}$, we have observed a broad and strong $\sigma^{*}\left(6 a_{1}\right)$ band below the Rydberg region and a weak shoulder arising from the $3 \mathrm{sa}_{1}$ Rydberg [4]. The $\sigma^{*}\left(6 a_{1}\right)$ and $3 s_{1}$ Rydberg orbitals are shown in Fig. 2, indicating that the $3 \mathrm{sa}_{1}$ orbital of the $\mathrm{CH}_{3}$ fragment is deformed by orthogonalization to the valence electrons of the $\mathrm{F}$ atom. There might be more or less RV mixing due to the avoided crossing for the same $\mathrm{a}_{1}$ symmetry. 
(a)
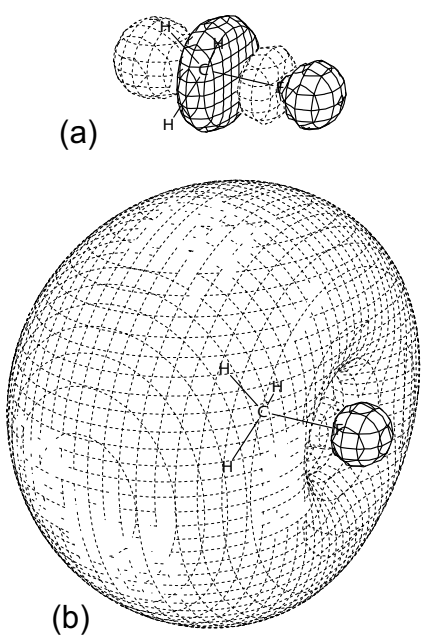

FIG. 2: Contour maps of (a) the $\sigma^{*}\left(6 \mathrm{a}_{1} *\right)$ orbital and (b) the $3 \mathrm{sa}_{1}$ Rydberg orbital in the $\mathrm{C} 1 \mathrm{~s}$ excitation of $\mathrm{CH}_{3} \mathrm{~F}$.

\section{VALENCE CONTRIBUTION IN CORE-TO-RYDBERG EXCITATION}

\section{A. $\mathrm{CH}_{4}$}

A simple molecular orbital picture predicts that the $\mathrm{CH}_{4}$ molecule has the $2 \mathrm{t}_{2} *$ and $3 \mathrm{a}_{1} *$ antibonding molecular orbitals. In the case of inner-shell excitation, the $\mathrm{C} 1 \mathrm{~s}\left(1 \mathrm{a}_{1}\right) \rightarrow 3 \mathrm{a}_{1}$ excitation is dipole forbidden but the $\mathrm{C} 1 \mathrm{~s}$ $\left(1 a_{1}\right) \rightarrow 2 t_{2}$ excitation is dipole allowed. However, no distinct $2 \mathrm{t}_{2} *$ resonance is observed in the $\mathrm{C} 1 \mathrm{~s}$ photoabsorption of $\mathrm{CH}_{4}$ [5]. The $\mathrm{C} 1 \mathrm{~s}$ photoabsorption spectrum shows many distinct Rydberg states, vibronically allowed $3 \mathrm{sa}_{1}$, strong $\mathrm{npt}_{2}$ series, and ndt 2 series. The Rydberg region in $\mathrm{CH}_{4}(\mathrm{C} 1 \mathrm{~s})$ [5] seems to be almost the same as in $\mathrm{CH}_{3} \mathrm{~F}(\mathrm{C} 1 \mathrm{~s})$ [4]. It is noticed that the $\mathrm{C} 1 \mathrm{~s}\left(1 \mathrm{a}_{1}\right) \rightarrow 3 \mathrm{pt}_{2}$ Rydberg excited state with three-fold degeneracy should be affected by Jahn-Teller (JT) distortion. Fig. 3 shows one of the three degenerate $3 \mathrm{pt}_{2} \mathrm{Ry}-$ dberg orbitals in $\mathrm{CH}_{4}$ in comparison with the $3 \mathrm{sa}_{1}$ Rydberg orbital. Considering that the $3 \mathrm{pt}_{2}$ orbital has no valence contribution and is purely atomic-like, we could expect a very weak JT distortion in the $3 \mathrm{pt}_{2}$ Rydberg state [6]. However, recent high-resolution $\mathrm{C}$ 1s photoabsorption spectra of $\mathrm{CH}_{4}[7,8]$ have shown that the $3 \mathrm{pt}_{2}$ Rydberg state shows strong vibrational contributions in addition to the total symmetric vibration. This vibrational enhancement should arise from the JT effect in $\mathrm{CH}_{4}$. Fig. 4 shows (b) a $\mathrm{C}_{3 \mathrm{v}}$-distorted 3p Rydberg orbital, together with (a) the $2 \mathrm{t}_{2} *$ orbital obtained without any diffuse function. The JT distorted $3 \mathrm{pt}_{2}$ orbital can get a large $2 \mathrm{t}_{2} *$ contribution. This valence mixing gives intensities to the vibronically excited states of the $\mathrm{C} 1 \mathrm{~s}\left(1 \mathrm{a}_{1}\right) \rightarrow 3 \mathrm{pt}_{2}$ excitation.
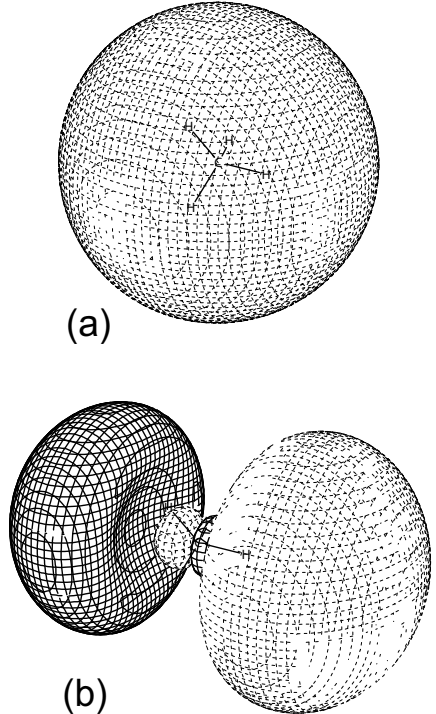

FIG. 3: Contour maps of (a) the 3sa $\mathrm{sa}_{1}$ Rydberg orbital and (b) one of the three $3 \mathrm{pt}_{2}$ Rydberg orbitals in the $\mathrm{C} 1 \mathrm{~s}$ excitation of $\mathrm{CH}_{4}$. The $3 \mathrm{pt}_{2}$ orbital has no valence contribution and is purely atomic-like.

(a)
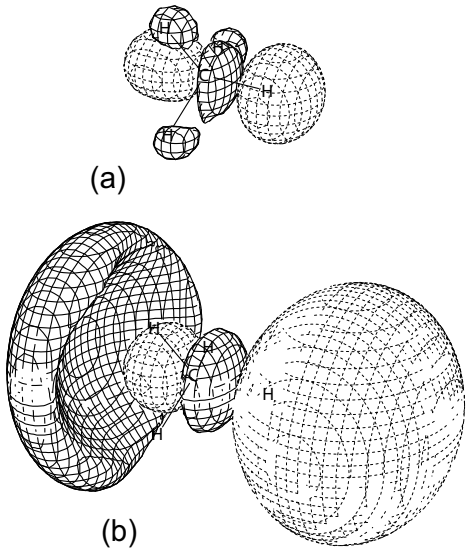

FIG. 4: Contour maps of (a) the $2 \mathrm{t}_{2} *$ orbital obtained without any diffuse function and (b) a $\mathrm{C}_{3 \mathrm{v}}$-distorted " $3 \mathrm{pt}_{2}$ " orbital in the $\mathrm{C} 1 \mathrm{~s}$ excitation of $\mathrm{CH}_{4}$. The $2 \mathrm{t}_{2} *$ valence mixing gives intensities to the vibronically excited states of the $\mathrm{C} 1 \mathrm{~s}\left(1 \mathrm{a}_{1}\right) \rightarrow 3 \mathrm{pt}_{2}$ excitation.

\section{B. $\mathrm{CO}_{2}(\mathrm{C} 1 \mathrm{~s})$}

In the $\mathrm{C} 1 \mathrm{~s}\left(2 \sigma_{g}\right)$ excitation spectrum of $\mathrm{CO}_{2}$, the strongest Rydberg state is of $3 \mathrm{~s} \sigma_{g}$ symmetry [8,9]. However, the C1s $\left(2 \sigma_{g}\right) \rightarrow 3 \mathrm{~s} \sigma_{g}$ Rydberg excitation is dipole-forbidden. Fig. 5 shows a contour map of the $3 \mathrm{~s} \sigma_{g}$ Rydberg orbital (Fig. 5(a)), which is mixed with the $5 \sigma_{g}{ }^{*}$ antibonding orbital (Fig. 5(b)). This valence mixing does not give any intensity from the $\mathrm{C} 1 \mathrm{~s}$ $\left(2 \sigma_{g}\right)$. On the other hand, Fig. 6 shows an orbital map for the same 3s Rydberg orbital at a bent geometry. The 3s Rydberg state has a large $\pi^{*}$ contribution. The $\mathrm{C} 1 \mathrm{~s}\left(2 \sigma_{g}\right) \rightarrow 2 \pi_{u} *$ valence excited state has a stable bent geometry due to the Renner-Teller effect on electronically degenerate states in linear polyatomic systems [10]. This bending geometry, or bend- 
ing motion in terms of molecular dynamics, is related to the vibronically induced $3 \mathrm{~s} \sigma_{g}$ Rydberg transition. In highresolution ARPIS spectra, bending vibrational fine structures are resolved in the $\mathrm{C} 1 \mathrm{~s}\left(2 \sigma_{g}\right) \rightarrow 3 \mathrm{~s} \sigma_{g}$ and $4 \mathrm{~s} \sigma_{g}$ Rydberg bands $[8,9]$.
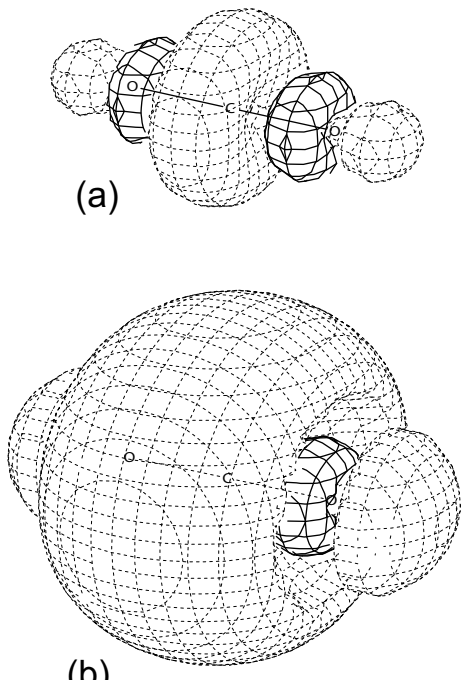

(b)

FIG. 5: Contour maps of (a) the $5 \sigma_{g}{ }^{*}$ valence orbital obtained without any diffuse function and (b) the $3 \mathrm{~s} \sigma_{g}$ Rydberg orbital in the $\mathrm{C} 1 \mathrm{~s}$ $\left(2 \sigma_{g}\right)$ excitation of $\mathrm{CO}_{2}$

(a)
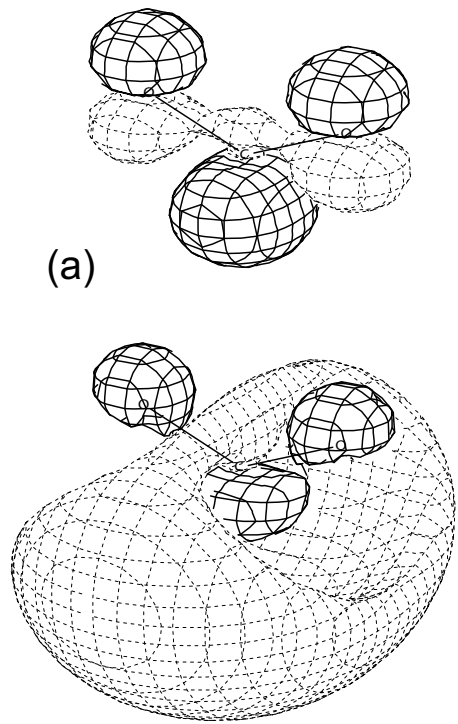

(b)

FIG. 6: Contour maps of (a) the $\pi^{*}$ orbital and (b) the 3s Rydberg orbital at a bent geometry in the $\mathrm{C} 1 \mathrm{~s}$ excitation of $\mathrm{CO}_{2}$. The $3 \mathrm{~s}$ Rydberg orbital has a large $\pi^{*}$ valence contribution.

\section{C. $\mathrm{N}_{2} \mathrm{O}$ and $\mathrm{CO}_{2}($ terminal $\mathrm{N}$ and $\mathrm{O})$}

$\mathrm{N}_{2} \mathrm{O}$ and $\mathrm{CO}_{2}$ are isoelectronic and the molecular orbitals look similar. The lowest $\sigma^{*}\left(8 \sigma^{*}\right)$ and 3s Rydberg orbitals excited from the central $\mathrm{N}\left(\mathrm{N}_{c}\right)$ are shown in Fig. 7. The orbital character is nearly the same as in $\mathrm{CO}_{2}(\mathrm{C} 1 \mathrm{~s})$ shown in Fig. 5. The $\mathrm{N}_{c}$ 1s excitation of $\mathrm{N}_{2} \mathrm{O}$ is expected to be similar to the $\mathrm{C} 1 \mathrm{~s}$ excitation of $\mathrm{CO}_{2}$. Unfortunately, the N1s excitation spectra include contributions from two $\mathrm{N} 1 \mathrm{~s}$ edges and do not show clear evidence for bending mode coupling in the $\mathrm{N}_{c}$ manifold [11]. On the other hand, the lowest $\sigma^{*}$ orbitals $\left(8 \sigma^{*}\right.$ of $\mathrm{N}_{2} \mathrm{O}$ in Fig. 7(a) and $5 \sigma_{g} *$ of $\mathrm{CO}_{2}$ in Fig. 5(a)) have a large po component on the terminal $\mathrm{N}\left(\mathrm{N}_{t}\right)$ and O. Fig. 8 shows a contour map of the " $4 \mathrm{~s}$ " Rydberg orbital excited from the $\mathrm{N}_{t} 1 \mathrm{~s}$. The $\mathrm{N}_{t} 4 \mathrm{~s}$ Rydberg orbital has a large $8 \sigma^{*}$ contribution with a p $\sigma$ component as shown in Fig. 7(a) and get its intensity from the $\mathrm{N}_{t} 1 \mathrm{~s} \rightarrow \mathrm{p} \sigma$ component $[1,11]$. Although the s-type Rydberg series is generally expected to be weak in the 1s photoabsorption, the Rydberg regions of the $\mathrm{N}_{t} 1 \mathrm{~s}$ and $\mathrm{O} 1 \mathrm{~s}$ excitations of $\mathrm{N}_{2} \mathrm{O}$ and $\mathrm{CO}_{2}$ are dominated in the observed spectra $[7,8,11]$ by the s-type Rydberg states $(4 \mathrm{~s}$ most dominant) due to the lowest $\sigma^{*}$ mixing with the p $\sigma$ component on the terminal atoms. However, it is noted that the $1 \mathrm{~s} \rightarrow 8 \sigma^{*}$ excitation of $\mathrm{N}_{2} \mathrm{O}$ and $1 \mathrm{~s} \rightarrow 5 \sigma_{g} *$ excitation of $\mathrm{CO}_{2}$ are not definitely identified as the $1 \mathrm{~s} \rightarrow 2 \mathrm{t}_{2} *$ of $\mathrm{CH}_{4}$. In these cases, it is reasonable to think that the valence contribution is dissolved in the Rydberg sea $[1,6,12]$.

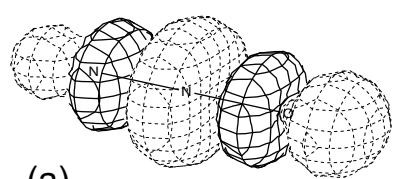

(a)

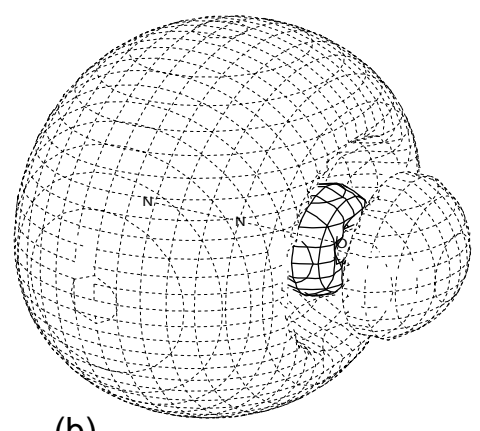

(b)

FIG. 7: Contour maps of (a) the lowest $\sigma^{*}\left(8 \sigma^{*}\right)$ orbital obtained without any diffuse function and (b) the RV-mixed $3 \mathrm{~s} \sigma$ orbitals in the central $\mathrm{N}\left(\mathrm{N}_{c}\right)$ 1s excitation of $\mathrm{N}_{2} \mathrm{O}$.

\section{SUMMARY}

Using molecular orbitals, we have discussed how the $1 \mathrm{~s} \rightarrow$ $\sigma^{*}$ excited state looks like in the $1 \mathrm{~s}$ excitation of $\mathrm{O}_{2}$ and $\mathrm{CH}_{3} \mathrm{~F}$, and how we know evidence for the $1 \mathrm{~s} \rightarrow \sigma^{*}$ contribution in 


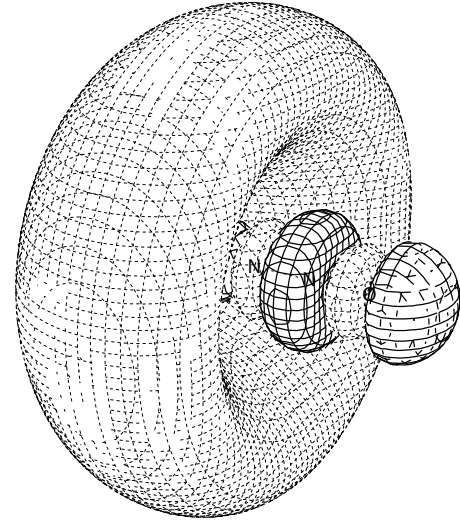

FIG. 8: A contour map of the RV-mixed $4 \mathrm{~s} \sigma$ orbital in the terminal $\mathrm{N}_{t}$ 1s excitation of $\mathrm{N}_{2} \mathrm{O}$.

photoabsorption spectra of $\mathrm{CH}_{4}, \mathrm{CO}_{2}$, and $\mathrm{N}_{2} \mathrm{O}$. In the former molecules, the excitation to the $\sigma^{*}$ orbital is identified in the Franck-Condon region from the ground state; in the latter molecules, the excitation to the $\sigma^{*}$ orbital is not clearly identified but its evidence is observed through the Rydberg-valence mixing in the Franck-Condon region. In $\mathrm{O}_{2}$, the $\sigma^{*}\left(3 \sigma_{u}{ }^{*}\right)$ orbital is mainly mixed with the $3 \mathrm{p} \sigma_{u}$ Rydberg orbital converging to the ${ }^{4} \Sigma^{+}\left({ }^{4} \Sigma_{g}^{+}\right)$ionization. In $\mathrm{CH}_{3} \mathrm{~F}$, the excitation to the $\sigma^{*}\left(6 \mathrm{a}_{1} *\right)$ orbital is observed below the $3 \mathrm{sa}_{1}$ Rydberg state. On the other hand, in $\mathrm{CH}_{4}$, the Jahn-Teller distortion of the $3 \mathrm{pt}_{2}$ Rydberg transition induces contribution from the $\sigma^{*}\left(2 t_{2} *\right)$ orbital component. In the $1 \mathrm{~s}$ excitations from the terminal atoms in $\mathrm{CO}_{2}$ and $\mathrm{N}_{2} \mathrm{O}$, some lower s-type Rydberg states get intensities from the $\sigma^{*}\left(5 \sigma_{g}^{*}\right.$ and $\left.8 \sigma^{*}\right)$ component. In the $1 \mathrm{~s}$ excitations from the central atoms in $\mathrm{CO}_{2}$ and $\mathrm{N}_{2} \mathrm{O}$, the $1 \mathrm{~s} \rightarrow \sigma^{*}$ excited state with gerade-gerade transition is a dipole-forbidden (dark) state (not exactly from $\mathrm{N}_{c}$ in $\mathrm{N}_{2} \mathrm{O}$ ). In $\mathrm{CO}_{2}$, the $\mathrm{C} 1 \mathrm{~s}$ excitation to the $3 \mathrm{~s} \sigma_{g}$ Rydberg orbital is vibronically enhanced through mixing with the $\mathrm{C} 1 \mathrm{~s}$ excitation to the $\pi^{*}\left(2 \pi_{u} *\right)$ orbital with the Renner-Teller effect.

\section{Acknowledgements}

The author acknowledges fruitful discussion with Dr. Miyabi Hiyama from a theoretical point of view and with Dr. Jun-ichi Adachi from an experimental point of view. The present work was partly supported by Grant-in-Aid for Scientific Research (B) from the Japan Society for the Promotion of Science (JSPS).
[1] N. Kosugi, J. Electron Spectrosc. 144-147, 1203 (2005).

[2] A. Yagishita, E. Shigemasa, and N. Kosugi, Phys. Rev. Lett. 72 3961 (1994).

[3] N. Kosugi, E. Shigemasa, and A. Yagishita, Chem. Phys. Lett. 190, 481 (1992).

[4] N. Kosugi, K. Ueda, Y. Shimizu, H. Chiba, M. Okunishi, K. Ohmori, Y. Sato, and E. Shigemasa, Chem. Phys. Lett. 246, 475 (1995).

[5] K. Ueda, M. Okunishi, H. Chiba, Y. Shimizu, K. Ohmori, Y. Sato, E. Shigemasa, and N. Kosugi, Chem. Phys. Lett. 236, 311 (1995).

[6] M. B. Robin, Higher Excited States of Polyatomic Molecules, Vol. III (Academic Press, New York, 1985) p.32, p.87.
[7] N. Kosugi, in Chemical Applications of Synchrotron Radiation, Part I, ed. T.-K. Sham (World Scientific, Singapore, 2002). p. 228 .

[8] N. Kosugi, J. Electron Spectrosc. 79, 351 (1996).

[9] J. Adachi, N. Kosugi, E. Shigemasa, and A. Yagishita, J. Phys. Chem. 100, 19783 (1996).

[10] J. Adachi, N. Kosugi, E. Shigemasa, and A. Yagishita, J. Chem. Phys. 107, 4919 (1997).

[11] J. Adachi, N. Kosugi, E. Shigemasa, and A. Yagishita, J. Chem. Phys. 102, 7369 (1995).

[12] R. J. Buenker and S. D. Peyerimhoff, Chem. Phys. Lett. 36, 415 (1975). 